\title{
Software Phase-Locked Loop based on Virtual Three- Phase for Power Grid
}

\author{
Junxiang Pei \\ Shanghai University of Engineering Science \\ Songjiang Shanghai \\ 201620, China
}

\author{
Hongtao Shan* \\ Corresponding Author \\ Shanghai University of Engineering Science \\ Songjiang Shanghai \\ 201620, China
}

\begin{abstract}
How to complete the phase-locked quickly and accurately is a basic key technology in the grid-connected inverter. Compared to the typical phase locking method with zero crossing, synchronous coordinate system software phaselocked loop (SPLL) has an advantage of easy to achieve, faster dynamic response, etc. This paper studied a strategy for applying the SPLL to the single phase grid-connected inverter. Firstly, use the virtual three-phase method to convert the single-phase voltage into three-phase balanced voltage. Then complete the software phase-locked with vector decoupling method in the synchronous coordinate system. Each simulation parameter of actual control system was calculated by tuning parameters of the regulator. The feasibility of this method is verified by experiment.
\end{abstract}

\section{Keywords}

Single-phase grid-connected, Virtual three-phase, Synchronous coordinate system, Software phase-locked loop (SPLL)

\section{INTRODUCTION}

In the systems such as: wind power integration converter, photovoltaic grid-connected inverter, static reactive power compensation device and so on, the information detection of power grid like real time phase frequency play an important role in the stability and following feature of the whole power grid. At present, the power grid environment is constantly changing worse, which put forward higher requirements to power grid inverter.

The fundamental of traditional zero crossing phase lock is to real-time detect the zero crossing point and frequency of the supply power. And then complete the phase lock based on the phase position detected before. This method is simple and easy to realize, but the dynamic response is unsatisfactory. Obviously it is a limit to the response of zero crossing method. But in contrast, the software phase-locked method could overcome the temperature drift of analog circuit effectively. It can not only complete the phase-lock precisely, but also obtain the amplitude and frequency of the power grid system. At present, the software phase-locked method has becoming one of the most important object of study in power electronics control area.

Synchronous coordinate system software phase-locked loop (SPLL) is the most popular software phase -locked method. This method can complete the phase lock quickly and precisely in three-phase balanced system, but the disadvantage is that it cannot be appropriate for single phase system. Aiming at the problem above, this paper approve a strategy for applying the SPLL to the single phase grid-connected inverter. The simulation model was built by using MATLAB/Simulink. Finally, the method is validated with an experimental platform.

\section{CONSTRUCTION METHOD OF VIRTUAL THREE-PHASE AND THE PRINCIPLE OF SPLL}

The fundamental of SPLL based on the synchronous rotating reference frame is that: Converting three-phase voltage to two-phase stationary reference frame firstly, then converting to two-phase voltage in dq rotating coordinate system. Finally, complete the phase-lock by comparing the phase difference to control.

As shown in Figure1. Assuming that the initial single phase voltage is $V_{a 1}$. In order to reduce the response time and improve the quick response ability of the system, this paper get the $V_{a 1}^{\prime}$ by means of delaying $-\frac{T}{6}$ of the phase $V_{a 1}^{\prime}$. It is easy to see the negative value of $V_{a 1}{ }^{\prime}$ is equal to $V_{a 1}{ }^{\prime}$. In the same way, this paper pick the negative value of $V_{a 1}{ }^{\prime}$ as $-V_{a 1}$ first, as shown in Figure1, $V_{a 2}=-V_{a 1}+V_{a 1}^{\prime}$. And now, this experiment has obtained the standard three-phase voltage $V_{a 1}{ }^{\prime}$, $V_{a 2}$ and $V_{a 3}$ by the method of virtual three-phase.

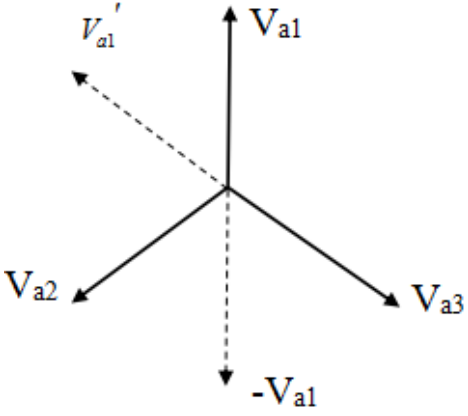

Fig1: Schematic of the virtual three phase voltage construction

After getting the virtual three-phase voltage, this paper could use the SPLL method to complete the phase lock experiment.

In synchronous reference frame, the phase relationship of the real voltage vector and phase-locked loop output voltage vector is shown in Figure2. $V$ represents the real voltage and $V_{p l l}$ represents the phase-locked loop output voltage. $V$ anticlockwise revolute at the angular velocity of $\omega$. The $d$ coordinates of synchronous reference frame coincides with $V_{p l l}$. Included angle between $\alpha$ coordinates and the real 
voltage vector $V$ is $\theta_{1}$, the phase-locked loop output voltage $V_{p l l}$ vector is $\theta_{2}$. Then make the phase of $V$ and $V_{p l l}$ approaching by PI control method. And when phase lock-loop complete phase lock, the two phase is equal to one another $\left(\theta_{1}=\theta_{2}\right) . V_{p l l}$ will departure from $V$ when the voltage of power grid suddenly changes. At this moment, necessary measures should be taken to keep the phase of PLL meet $\theta_{1}=\theta_{2}$.

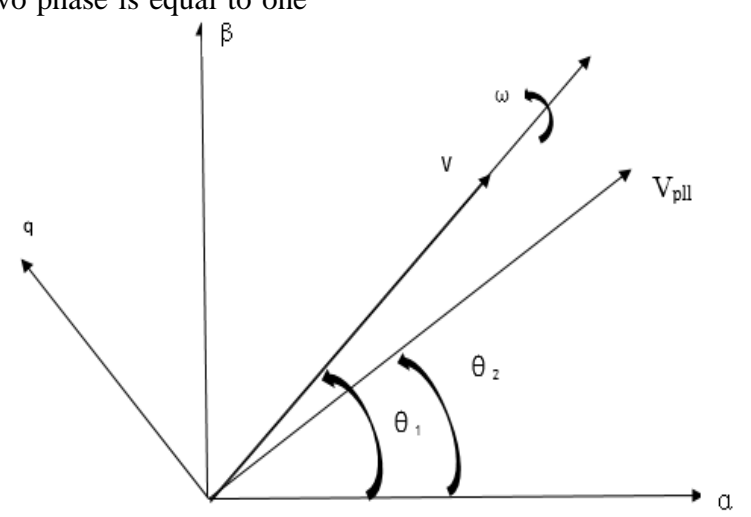

Fig2: Single phase synchronous coordinate system and the voltage vector

\section{DESIGN OF SYNCHRONOUS COORDINATE SYSTEM SPLL BASED ON THE VIRTUAL THREE PHASE VOLTAGE}

The schematic of synchronous coordinate system SPLL is shown in Figure 3. Firstly, this paper get virtual three-phase voltage $\mathrm{V}_{\mathrm{a} 1}, \mathrm{~V}_{\mathrm{a} 2}, \mathrm{~V}_{\mathrm{a} 3}$ through the method above. And then transform the voltage $\mathrm{V}_{\mathrm{a} 1}, \mathrm{~V}_{\mathrm{a} 2}, \mathrm{~V}_{\mathrm{a} 3}$ to $V_{d}$ and $V_{q}$ in synchronous rotational coordinates. In order to make $V_{q}$

equal to zero, this paper bring PI controller and integral element into the feedback circuit. Keep adjusting the skewing $\theta$ until SPLL complete the phase lock precisely.

Next step work is: analyze the process of voltage converting according to the Figure 3 control block above. Assuming that the initial single-phase voltage is A phase and its value is zero. The virtual three-phase voltage we got through A phase could be expressed as below.

$$
\left[\begin{array}{c}
V_{a 1}(t) \\
V_{a 2}(t) \\
V_{a 3}(t)
\end{array}\right]=V\left[\begin{array}{c}
\cos (\omega t) \\
\cos \left(\omega t-\frac{2}{3} \pi\right) \\
\cos \left(\omega t+\frac{2}{3} \pi\right)
\end{array}\right]
$$

Clark transform. It transforms the virtual three-phase voltage into a two-phase voltage of static coordinate system. As is shown below:

$\left[\begin{array}{l}V_{\alpha} \\ V_{\beta}\end{array}\right]=\frac{2}{3}\left[\begin{array}{rrr}1 & -\frac{1}{2} & -\frac{1}{2} \\ 0 & \frac{\sqrt{3}}{2} & \frac{\sqrt{3}}{2}\end{array}\right]\left[\begin{array}{c}V_{a 1}(t) \\ V_{a 2}(t) \\ V_{a 3}(t)\end{array}\right]=V\left[\begin{array}{l}\cos (\omega t) \\ \sin (\omega t)\end{array}\right]$

The next step is Park transform, which transforms the twophase voltage $V_{\alpha}$ and $V_{\beta}$ into $V_{d}$ and $V_{q}$ of synchronous rotating frame. As is shown below:

$\left[\begin{array}{l}V_{d} \\ V_{q}\end{array}\right]=\left[\begin{array}{cc}\cos \theta_{o} & \sin \theta_{o} \\ -\sin \theta_{o} & \cos \theta_{o}\end{array}\right]\left[\begin{array}{c}V_{\alpha} \\ V_{\beta}\end{array}\right]=V\left[\begin{array}{c}\cos \left(\omega t-\theta_{o}\right) \\ \sin \left(\omega t-\theta_{o}\right)\end{array}\right]$

It is not hard to see from the third formula that if the phase lock is uncompleted $\left(\omega t \neq \theta_{o}\right)$, $\omega \mathrm{t}$ will not equal to $\theta_{0}$. But as long as they equal to each other $\left(\omega t=\theta_{o}\right), V_{d}=U$, $V_{q}=0$.The $S P L L$ will turn to phase-lock completed state. Now it is confirmed that a proper choice of SPLL parameter is the key to phase-lock success. Therefore, the simulation experiment is successful.

The first step of rotational coordinate system transformation is

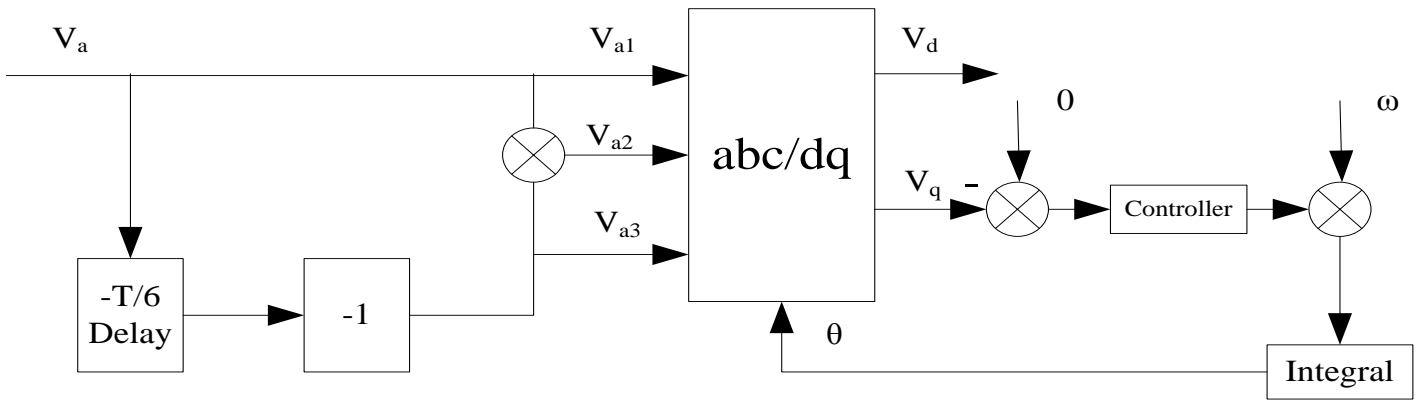

Fig3: Control block of synchronous coordinate system $S P L L$ based on the virtual three-phase voltage 


\section{SIMULATION EXPERIMENT \\ RESULT}

In order to test the precision and dynamic response ability of SPLL method, this paper set up a simulation model in the MATLAB software. Below is the initial parameters table.
Figure 4 is SPLL simulation model. Figure 5 is the virtual three-phase voltage simulation model. The input voltage of Figure 4 is the virtual three-phase voltage got in Figure 5.

Table 1. System simulation parameters

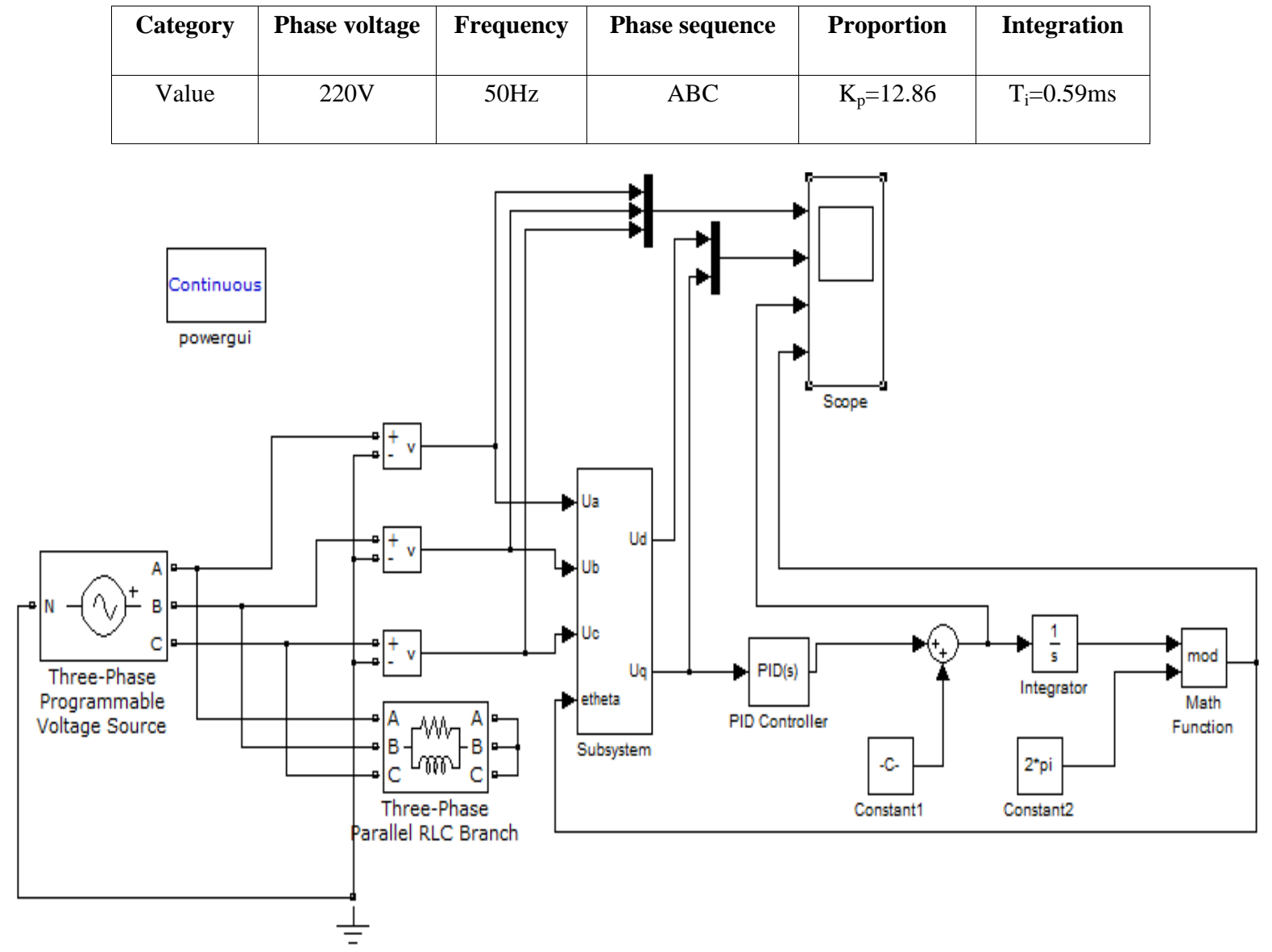

Fig4: SPLL simulation model

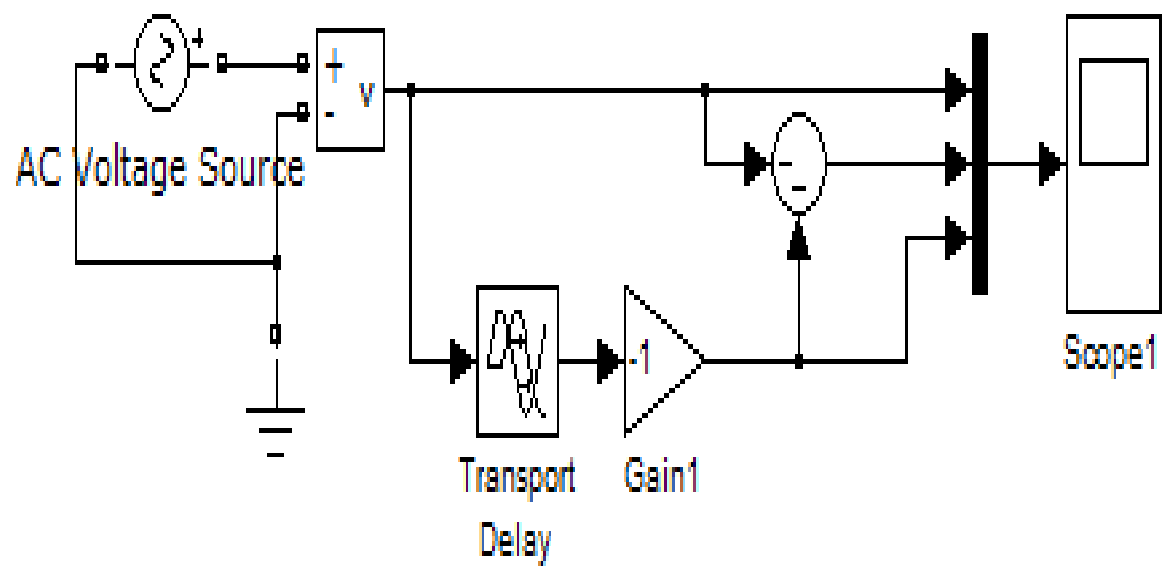

Fig5: virtual three-phase simulation model 


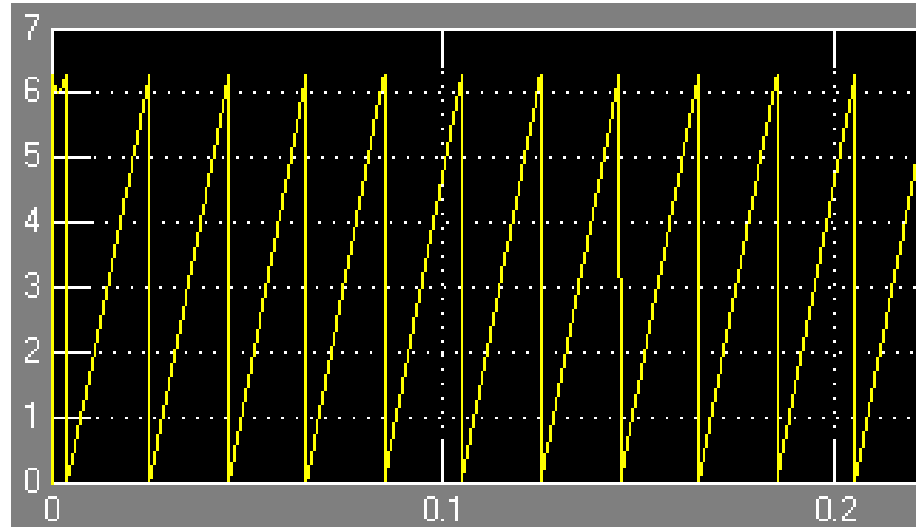

Fig6(a):phase-locked loop simulation waveform

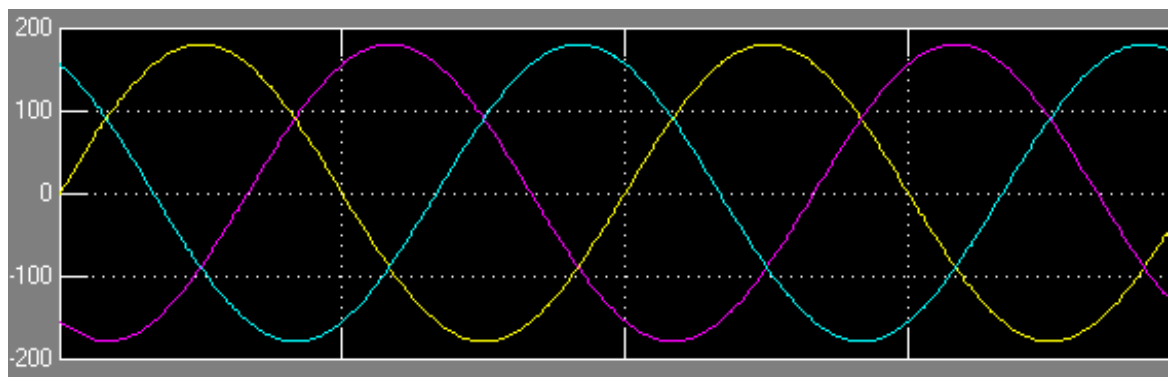

Fig6(b):three-phase inverter voltage

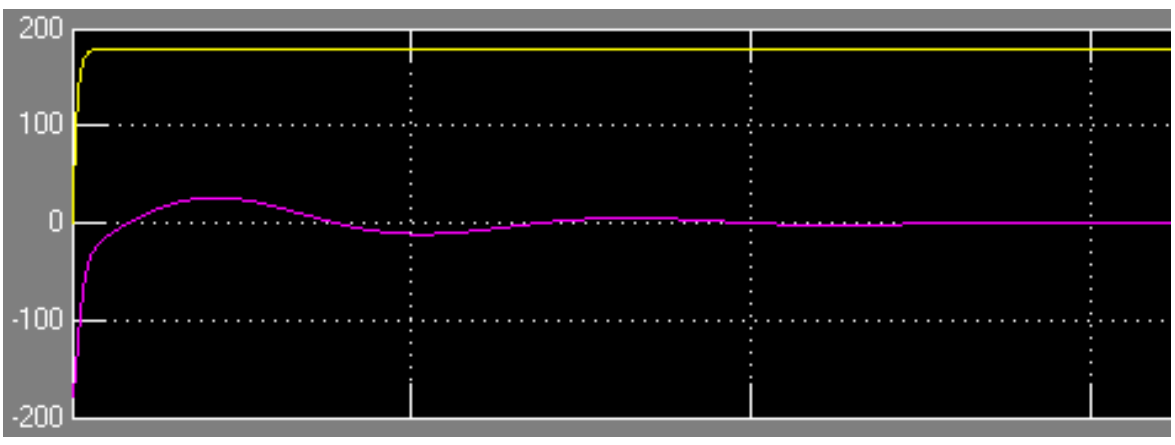

Fig6(c): $V_{d} V_{q}$ voltage waveform in the rotating coordinates

The simulation waveform of whole simulation model is shown in Figure 6. Get the virtual three-phase voltage first, which is shown in Figure 6 (a).It is not hard to see that the phase angle of inverter voltage is up to standard. And the phase lock time is nearly $2 \mathrm{~ms}$.It is more quickly than popular used phase-locked of passing zero. Figure 6 (b) is the output voltage phase angle of SPLL. It shows that the phase-lock precision is higher than zero crossing phase-lock method. The inverter voltage is close to $220 \mathrm{~V}$ and the error is within the scope of permit. The Figure 6 (c) shows that $V_{q}$ voltage component can approach to zero quickly. $V_{d}$ is the inverter voltage in synchronous rotational coordinates. And when the value of $V_{q}$ equals to zero, the phase-lock is completed.

\section{EXPERIMENT RESULT}

After accomplishing the simulation experiment, which confirm the feasibility of SPLL method. This paper carry out SPLL experiment in the sample machine further. The main experimental waveform is shown in Figure 7. The phase angles of mains voltage and inverter output voltage are nearly equal within bounds. Every block represents one millisecond. And the delay of SPLL method is about 3.3 millisecond. It is obviously more quickly than zero crossing phase-lock method. And the inverter output voltage is nearly $218 \mathrm{~V}$, the error is within scope of permit. So it is confirmed that this method is successful.

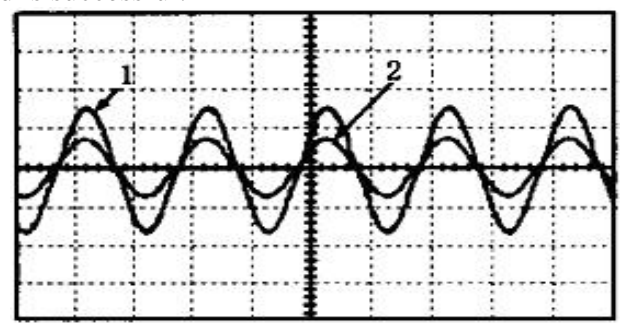

1- mains voltage

2- inverter voltage

Fig7: $S P L L$ experiment waveform

\section{CONCLUSION}

This paper verify the feasibility of SPLL method by simulation and experiment in sample machine. The algorithm of SPLL is simple and easy to achieve. SPLL method has excellent dynamic ability by making use of the internal relations of balanced three-phase voltage. 


\section{REFERENCES}

[1] Zhao Chunjiang, Yang Jinhuan and Chen Zhonghua, "State \& Development of Photovoltaic Application", Energy Conservation Technology., Vol. 25, No. 145, 2007, pp. 461-465.

[2] Li Jinguo and Jin Xinmin, "Design Control System for a Low-power Photovoltaic Inverter Interconnected with Electric Utility Circuit", Power Supply Technologies and Applications, Vol. 5, No. 12, 2002, pp. 655-658.

[3] Bao Lai, Lin Chengwu and Cheng Limin,"Research on Grid-connected Inverter based on Current Track Control in Wind Generation System", Power Electronics., Vol. 42, No. 6, 2008, pp. 53-55.

[4] Liu Haisheng and Tong Shizhong,"Design of Single Phrase Photovoltaic Grid-connected Inverter with Medium Power Rating", The World of Power Supply., No. 4, 2008, pp. 49-52.

[5] Wang Yu, "Research on control Strategies for Double Fed Wind Power Generation System", Master thesis, Univ. of Tianjin, China, 2009.

[6] Wang Zhaoan and Huang Jun "power electronics", China Machine Press., No. 4, 2008, pp. 163-169.

[7] Liu Xiangdong "Principles and Applications of DSP technology", China Electric Power Press., No. 5, 2007, pp. 220-235.

[8] Liu Heping "TMS320LF240xDSP structure, principle and application", Beijing Aeronautics and Astronautics Press., No. 9, 2002, pp. 10-20.

[9] Y. C. Kuo, T. J. Liang and J. F. Chen , "Novel Maximum-Power-Point-Tracking Controller for Photovoltaic Energy Conversion System", IEEE Transactions On Industrial Electronics , vol. 48 , pp.594 -
601,2001

[10] T. Markvart and L. Castafier , Practical Handbook of Photovoltaics: Fundamentalsand Applications, 2003 :Elsevier

[11] C. Hua and J. R. Lin , "DSP-Based Controller Application in Battery Storage of Photovoltaic System" , in IEEE 22nd International Conference on Industrial Electronics, Control, and Instrumentation (IECON '96), 1996, pp. $1705-1710$

[12] M. Jang, M. Ciobotaru and V. G. Agelidis , "A SinglePhase Grid-Connected Fuel Cell System Based on a Boost-Inverter" , IEEE Transactions on Power Electronics , vol. 2 , no. 1 , pp.279 -288 , 2013

[13] R. O. Caceres and I. Barbi , "A Boost DC-AC Converter: Analysis,Design, and Experimentation" , IEEE Transactions on Power Electronics , vol. 14, 1999

[14] P. Sanchis, A. Ursa, E. Gubia and L. Marroyo , "Boost DC-AC Inverter: A New Control Strategy" , IEEE Transactions On Power Electronics, vol. 20 , no. 2, pp.343 -353, 2005

[15] C. Albea and F. Gordillo , "Control of the Boost DC-AC Converter with RL Load by Energy Shaping" , in Proceedings of the 46th IEEE Conference on Decision and Control , pp.2417 -2422

[16] T. Esram , "Comparison of Photovoltaic Array Maximum Power Point Tracking Techniques" , IEEE Transactions On Energy Conversion , vol. 22 , pp.439 449,2007

[17] K. Jha, S. Mishra and A. Joshi , "High-Quality Sine Wave Generation Using a Differential Boost Inverter at Higher Operation Frequency" , IEEE Transactions on Industry Applications , pp.1 -14, 2013 